PROCEEDINGS OF THE

AMERICAN MATHEMATICAL SOCIETY

Volume 132, Number 5, Pages 1295-1303

S 0002-9939(03)07185-5

Article electronically published on December 5, 2003

\title{
A NOTE ON PERIODIC SOLUTIONS OF NONAUTONOMOUS SECOND-ORDER SYSTEMS
}

\author{
CHUN-LEI TANG AND XING-PING WU \\ (Communicated by Jonathan M. Borwein)
}

\begin{abstract}
A multiplicity theorem is obtained for periodic solutions of nonautonomous second-order systems with partially periodic potentials by the minimax methods.
\end{abstract}

\section{Introduction AND MAIN RESUlts}

Consider the second-order systems

$$
\left\{\begin{array}{l}
\ddot{u}(t)+\nabla F(t, u(t))=e(t) \\
u(0)-u(T)=\dot{u}(0)-\dot{u}(T)=0
\end{array} \quad \text { a.e. } t \in[0, T]\right.
$$

where $T>0$ and $F:[0, T] \times R^{N} \rightarrow R$ satisfies the following assumption:

$F(t, x)$ is measurable in $t$ for every $x \in R^{N}$ and continuously differentiable in $x$ for a.e. $t \in[0, T]$, and there exist $f, g \in L^{1}\left(0, T ; R^{+}\right)$and $\alpha \in[0,1[$ such that

$$
|F(t, 0)|+|\nabla F(t, x)| \leq f(t)|x|^{\alpha}+g(t)
$$

for all $x \in R^{N}$ and a.e. $t \in[0, T]$. Suppose that $F(t, x)$ is $T_{i}$-periodic in $x_{i}, 1 \leq i \leq r$, that is,

$$
F\left(t, x+\sum_{i=1}^{r} k_{i} T_{i} e_{i}\right)=F(t, x)
$$

for a.e. $t \in[0, T]$, all $x \in R^{N}$ and all integers $k_{i}, 1 \leq i \leq r$, where $\left(e_{i}\right)(1 \leq i \leq N)$ is the canonical basis of $R^{N}$.

With periodic potentials, that is, (3) holding with $r=N$, the existence and multiplicity theorems are obtained for the nonautonomous second-order system (1) in [1] and [2] respectively. Note that (2) holds automatically with $\alpha=0$ in this case. Under the condition (2) with $\alpha=0,3$ and 4 consider the nonautonomous secondorder system (1) with partially periodic (that is, (3) holding with $0 \leq r \leq N$ ) and partially uniformly coercive potentials $\left(F(t, x) \rightarrow+\infty\right.$ for every $\left(x_{1}, \ldots, x_{r}\right) \in R^{r}$ as $\left(x_{r+1}, \ldots, x_{N}\right)$ tends to infinity in $\left.R^{N-r}\right)$. Recently [5] obtains the same result

Received by the editors April 29, 2001.

2000 Mathematics Subject Classification. Primary 34C25, 47N20, 58E50.

Key words and phrases. Periodic solution, second-order system, periodicity, Sobolev's inequality, Wirtinger's inequality, the minimax methods.

Supported by National Natural Science Foundation of China, by Major Project of Science and Technology of MOE, P.R.C. and by the Teaching and Research Award program for Outstanding Young Teachers in Higher Education Institutions of MOE, P.R.C.

(C)2003 American Mathematical Society 
as [4 by replacing the partially uniformly coercive condition with the partially semicoercive condition (that is, $\int_{0}^{T} F(t, x) d t \rightarrow+\infty$ for every $\left(x_{1}, \ldots, x_{r}\right) \in R^{r}$ as $\left(x_{r+1}, \ldots, x_{N}\right)$ tends to infinity in $\left.R^{N-r}\right)$.

In this paper we obtain the same result as [4] and [5] but under weaker coercivity conditions. In fact, we consider the nonautonomous second-order system (1) with the partially periodic potential and sublinear nonlinearity (that is, (2) holding with $0 \leq \alpha<1$ ), which is motivated by [5] and [6]. Some results mentioned above are unified and generalized. The following main results are obtained by the minimax methods.

Theorem. Suppose that (3) holds and $e \in L^{1}\left(0, T ; R^{N}\right)$ satisfying

$$
\int_{0}^{T} e(t) d t=0
$$

Assume that (2) holds and

$$
|x|^{-2 \alpha} \int_{0}^{T} F(t, x) d t \rightarrow+\infty(\text { or }-\infty)
$$

as $x$ tends to infinity in $0 \times R^{N-r}$. Then problem (1) has at least $r+1$ geometrically distinct solutions in $H_{T}^{1}$, where

$$
H_{T}^{1}=\left\{\begin{array}{l|l}
u:[0, T] \rightarrow R^{N} & \begin{array}{l}
u \text { is absolutely continuous, } \\
u(0)=u(T) \text { and } \dot{u} \in L^{2}\left(0, T ; R^{N}\right)
\end{array}
\end{array}\right\}
$$

is a Hilbert space with the norm given by

$$
\|u\|=\left(\int_{0}^{T}|u(t)|^{2} d t+\int_{0}^{T}|\dot{u}(t)|^{2} d t\right)^{\frac{1}{2}}
$$

for $u \in H_{T}^{1}$.

Corollary. Suppose that $F(t, x)$ is measurable in $t$ for every $x \in R^{N}$ and continuously differentiable in $x$ for a.e. $t \in[0, T]$, and there exists $g \in L^{1}\left(0, T ; R^{+}\right)$such that

$$
|F(t, 0)|+|\nabla F(t, x)| \leq g(t)
$$

for all $x \in R^{N}$ and a.e. $t \in[0, T]$ and

$$
\int_{0}^{T} F(t, x) d t \rightarrow+\infty(\text { or }-\infty)
$$

as $x$ tends to infinity in $0 \times R^{N-r}$. Assume that (3) and (4) hold. Then problem (1) has at least $r+1$ geometrically distinct solutions in $H_{T}^{1}$.

Remark. Our Theorem unifies and generalizes Theorems 1-3 in [5] and Theorems 1-2 in [6], which are the special cases of our Theorem corresponding to $\alpha=0$ and $r=0$ respectively. Thus Theorem 2.1 in [4], Theorems 1.5, 1.6 and 4.8 in [7] and Theorem 0.3 in [2] all are the corollaries of our Theorem. There are functions $F$ satisfying our Theorem and not satisfying the results in [1]-[10]. For example, let $0<\alpha<1$ and

$$
F(t, x)=\beta(t)\left(r+1+\sin x_{1}+\cdots+\sin x_{r}+\sum_{j=r+1}^{N}\left|x_{j}\right|^{2}\right)^{\frac{\alpha+1}{2}}+(e(t), x),
$$


where $x=\left(x_{1}, x_{2}, \ldots, x_{N}\right) \in R^{N}, \beta \in L^{1}(0, T)$ with

$$
\int_{0}^{T} \beta(t) d t \neq 0
$$

and $e \in L^{1}\left(0, T ; R^{N}\right)$ satisfying (4).

\section{Proof of THEOREM}

For $u \in H_{T}^{1}$, let $\bar{u}=\frac{1}{T} \int_{0}^{T} u(t) d t$ and $\widetilde{u}(t)=u(t)-\bar{u}$. Then we have Sobolev's inequality

$$
\|\widetilde{u}\|_{\infty}^{2} \leq \frac{T}{12}\|\dot{u}\|_{L^{2}}^{2}
$$

and Wirtinger's inequality

$$
\|\widetilde{u}\|_{L^{2}} \leq \frac{T}{2 \pi}\|\dot{u}\|_{L^{2}}
$$

for all $u \in H_{T}^{1}$ (see Proposition 1.3 in [7]). Put $\hat{u}(t)=P \bar{u}+Q \bar{u}+\widetilde{u}(t)$, where

$$
P \bar{u}=\sum_{i=r+1}^{N}\left(\bar{u}, e_{i}\right) e_{i}, \quad Q \bar{u}=\sum_{i=1}^{r}\left[\left(\bar{u}, e_{i}\right)-l_{i} T_{i}\right] e_{i},
$$

and $l_{i}(1 \leq i \leq r)$ is the unique integer such that

$$
0 \leq\left(\bar{u}, e_{i}\right)-l_{i} T_{i}<T_{i} .
$$

Define the functional $\varphi$ on $H_{T}^{1}$ by

$$
\varphi(u)=\frac{1}{2} \int_{0}^{T}|\dot{u}(t)|^{2} d t-\int_{0}^{T} F(t, u(t)) d t+\int_{0}^{T}(e(t), u(t)) d t .
$$

Then $\varphi$ is continuously differentiable by Theorem 1.4 in [7, because (2) implies that

$$
|F(t, x)| \leq|F(t, 0)|+\left|\int_{0}^{1}(\nabla F(t, s x), x) d s\right| \leq f(t)|x|^{\alpha+1}+(|x|+1) g(t)
$$

for a.e. $t \in[0, T]$ and all $x \in R^{N}$. Moreover, one has

$$
\left\langle\varphi^{\prime}(u), v\right\rangle=\int_{0}^{T}(\dot{u}(t), \dot{v}(t)) d t-\int_{0}^{T}(\nabla F(t, u(t)), v(t)) d t+\int_{0}^{T}(e(t), v(t)) d t
$$

for all $u, v \in H_{T}^{1}$.

Let

$$
G=\left\{\sum_{i=1}^{r} k_{i} T_{i} e_{i} \mid k_{i} \text { is integer, } 1 \leq i \leq r\right\}
$$

be a discrete subgroup of $H_{T}^{1}$ and let $\pi: H_{T}^{1} \rightarrow H_{T}^{1} / G$ be the canonical surjection. It is obvious that $H_{T}^{1} / G=X \times V$, where $X=Y+Z, Y=\widetilde{H}_{T}^{1}=\left\{u \in H_{T}^{1} \mid \bar{u}=\right.$ $0\}, Z=\operatorname{span}\left\{e_{r+1}, \ldots, e_{N}\right\}$ and $V=\operatorname{span}\left\{e_{1}, \ldots, e_{r}\right\} / G$ is isomorphic to the torus $T^{r}$. Define $\psi: X \times V \rightarrow R$ by

$$
\psi(\pi(u))=\varphi(u) .
$$

It follows from (3) that $\psi$ is well-defined. Moreover, $\psi$ is continuously differentiable.

Now we begin to prove our main result. 
Proof of Theorem. In the case that $\left(5^{+}\right)$holds, the proof relies on the generalized saddle point theorem due to Liu [4. First assume that $\left(\pi\left(u_{n}\right)\right)$ is a (PS) sequence for $\psi$, that is, $\psi\left(\pi\left(u_{n}\right)\right)$ is bounded and $\psi^{\prime}\left(\pi\left(u_{n}\right)\right) \rightarrow 0$. Then $\varphi\left(u_{n}\right)$ is bounded and $\varphi^{\prime}\left(u_{n}\right) \rightarrow 0$. It follows from (2) and Sobolev's inequality (6) that

$$
\begin{aligned}
\mid \int_{0}^{T}( & \nabla F(t, \hat{u}(t)), \widetilde{u}(t)) d t|=| \int_{0}^{T}(\nabla F(t, Q \bar{u}+P \bar{u}+\widetilde{u}(t)), \widetilde{u}(t)) d t \mid \\
\leq & \int_{0}^{T} f(t)|Q \bar{u}+P \bar{u}+\widetilde{u}(t)|^{\alpha}|\widetilde{u}(t)| d t+\int_{0}^{T} g(t)|\widetilde{u}(t)| d t \\
\leq & \int_{0}^{T} 2 f(t)\left(|Q \bar{u}+P \bar{u}|^{\alpha}+|\widetilde{u}(t)|^{\alpha}\right)|\widetilde{u}(t)| d t+\int_{0}^{T} g(t)|\widetilde{u}(t)| d t \\
\leq & 2\left(|Q \bar{u}+P \bar{u}|^{\alpha}+\|\widetilde{u}\|_{\infty}^{\alpha}\right)\|\widetilde{u}\|_{\infty} \int_{0}^{T} f(t) d t+\|\widetilde{u}\|_{\infty} \int_{0}^{T} g(t) d t \\
\leq & \frac{3}{T}\|\widetilde{u}\|_{\infty}^{2}+\frac{T}{3}|Q \bar{u}+P \bar{u}|^{2 \alpha}\left(\int_{0}^{T} f(t) d t\right)^{2} \\
& +2\|\widetilde{u}\|_{\infty}^{\alpha+1} \int_{0}^{T} f(t) d t+\|\widetilde{u}\|_{\infty} \int_{0}^{T} g(t) d t \\
\leq & \frac{1}{4} \int_{0}^{T}|\dot{u}(t)|^{2} d t+C_{1}\left(|P \bar{u}|^{2 \alpha}+1\right) \\
& +C_{2}\left(\int_{0}^{T}|\dot{u}(t)|^{2} d t\right)^{\frac{\alpha+1}{2}}+C_{3}\left(\int_{0}^{T}|\dot{u}(t)|^{2} d t\right)^{\frac{1}{2}}
\end{aligned}
$$

for all $u \in H_{T}^{1}$ and some positive constants $C_{1}, C_{2}$ and $C_{3}$, where we have made use of the fact that $|Q \bar{u}|$ is bounded. Hence one has

$$
\begin{aligned}
\left\|\widetilde{u}_{n}\right\| \geq & \left|\left\langle\varphi^{\prime}\left(u_{n}\right), \widetilde{u}_{n}\right\rangle\right| \\
= & \left|\left\langle\varphi^{\prime}\left(\hat{u}_{n}\right), \widetilde{u}_{n}\right\rangle\right| \\
= & \left.\left|\int_{0}^{T}\right| \dot{u}_{n}(t)\right|^{2} d t-\int_{0}^{T}\left(\nabla F\left(t, \hat{u}_{n}(t)\right), \widetilde{u}_{n}(t)\right) d t+\int_{0}^{T}\left(e(t), \widetilde{u}_{n}(t)\right) d t \mid \\
\geq & \frac{3}{4} \int_{0}^{T}\left|\dot{u}_{n}(t)\right|^{2} d t-C_{1}\left(\left|P \bar{u}_{n}\right|^{2 \alpha}+1\right)-C_{2}\left(\int_{0}^{T}\left|\dot{u}_{n}(t)\right|^{2} d t\right)^{\frac{\alpha+1}{2}} \\
& -C_{3}\left(\int_{0}^{T}\left|\dot{u}_{n}(t)\right|^{2} d t\right)^{\frac{1}{2}}-\left\|\widetilde{u}_{n}\right\|_{\infty} \int_{0}^{T}|e(t)| d t \\
\geq & \frac{3}{4} \int_{0}^{T}\left|\dot{u}_{n}(t)\right|^{2} d t-C_{1}\left(\left|P \bar{u}_{n}\right|^{2 \alpha}+1\right)-C_{2}\left(\int_{0}^{T}\left|\dot{u}_{n}(t)\right|^{2} d t\right)^{\frac{\alpha+1}{2}} \\
& -C_{4}\left(\int_{0}^{T}\left|\dot{u}_{n}(t)\right|^{2} d t\right)^{\frac{1}{2}}
\end{aligned}
$$


for large $n$ and positive constant $C_{4}$ by the fact that $\varphi^{\prime}\left(u_{n}\right) \rightarrow 0,(4)$ and Sobolev's inequality. It follows from Wirtinger's inequality (7) that

$$
\begin{aligned}
\left(1+\frac{T^{2}}{4 \pi^{2}}\right)^{\frac{1}{2}}\left(\int_{0}^{T}\left|\dot{u}_{n}(t)\right|^{2} d t\right)^{\frac{1}{2}} & \geq\left\|\widetilde{u}_{n}\right\| \\
& \geq \frac{3}{4} \int_{0}^{T}\left|\dot{u}_{n}(t)\right|^{2} d t-C_{1}\left(\left|P \bar{u}_{n}\right|^{2 \alpha}+1\right) \\
& -C_{2}\left(\int_{0}^{T}\left|\dot{u}_{n}(t)\right|^{2} d t\right)^{\frac{\alpha+1}{2}}-C_{4}\left(\int_{0}^{T}\left|\dot{u}_{n}(t)\right|^{2} d t\right)^{\frac{1}{2}}
\end{aligned}
$$

for large $n$, which implies that

$$
C\left|P \bar{u}_{n}\right|^{\alpha} \geq\left(\int_{0}^{T}\left|\dot{u}_{n}(t)\right|^{2} d t\right)^{\frac{1}{2}}-C_{5}
$$

for some $C>0, C_{5}>0$ and all large $n$. By (2) and Sobolev's inequality (6) we have

$$
\begin{aligned}
\mid \int_{0}^{T}[F & (t, \hat{u}(t))-F(t, P \bar{u})] d t \mid \\
= & \left|\int_{0}^{T} \int_{0}^{1}(\nabla F(t, P \bar{u}+s(Q \bar{u}+\widetilde{u}(t))), Q \bar{u}+\widetilde{u}(t)) d s d t\right| \\
\leq & \int_{0}^{T} \int_{0}^{1} f(t)|P \bar{u}+s(Q \bar{u}+\widetilde{u}(t))|^{\alpha}|Q \bar{u}+\widetilde{u}(t)| d s d t \\
& +\int_{0}^{T} g(t)|Q \bar{u}+\widetilde{u}(t)| d t \\
\leq & \int_{0}^{T} 2 f(t)\left(|P \bar{u}|^{\alpha}+|Q \bar{u}+\widetilde{u}(t)|^{\alpha}\right)|Q \bar{u}+\widetilde{u}(t)| d t \\
& +\int_{0}^{T} g(t)|Q \bar{u}+\widetilde{u}(t)| d t \\
\leq & 2\left(|P \bar{u}|^{\alpha}+\left(|Q \bar{u}|^{2}+\|\widetilde{u}\|_{\infty}\right)^{\alpha}\right)\left(|Q \bar{u}|+\|\widetilde{u}\|_{\infty}\right) \int_{0}^{T} f(t) d t \\
& +\left(\mid Q \bar{u}+\|\widetilde{u}\|_{\infty}\right) \int_{0}^{T} g(t) d t \\
\leq & \frac{3}{T}\left(|Q \bar{u}|+\|\widetilde{u}\|_{\infty}\right)^{2}+\frac{T}{3}|P \bar{u}|^{2 \alpha}\left(\int_{0}^{T} f(t) d t\right)^{2} \\
& +2\left(|Q \bar{u}|+\|\widetilde{u}\|_{\infty}\right)^{\alpha+1} \int_{0}^{T} f(t) d t+\left(|Q \bar{u}|+\|\widetilde{u}\|_{\infty}\right) \int_{0}^{T} g(t) d t \\
\leq & \frac{1}{4} \int_{0}^{T}|\dot{u}(t)|^{2} d t+C_{6}\left(|P \bar{u}|^{2 \alpha}+1\right) \\
& +C_{7}\left(\int_{0}^{T}|\dot{u}(t)|^{2} d t\right)^{\frac{\alpha+1}{2}}+C_{8}\left(\int_{0}^{T}|\dot{u}(t)|^{2} d t\right)^{\frac{1}{2}}
\end{aligned}
$$


for all $u \in H_{T}^{1}$ and some positive constants $C_{6}, C_{7}$ and $C_{8}$. It follows from the boundedness of $\left\{\varphi\left(u_{n}\right)\right\},(8),(4),(9)$ and Sobolev's inequality (6) that

$$
\begin{aligned}
C_{9} \leq & \varphi\left(u_{n}\right) \\
= & \varphi\left(\hat{u}_{n}\right) \\
= & \frac{1}{2} \int_{0}^{T}\left|\dot{u}_{n}(t)\right|^{2} d t-\int_{0}^{T}\left[F\left(t, \hat{u}_{n}(t)\right)-F\left(t, P \bar{u}_{n}\right)\right] d t \\
& -\int_{0}^{T} F\left(t, P \bar{u}_{n}\right) d t+\int_{0}^{T}\left(e(t), \widetilde{u}_{n}(t)\right) d t \\
\leq & \frac{3}{4} \int_{0}^{T}\left|\dot{u}_{n}(t)\right|^{2} d t+C_{6}\left(\left|\bar{u}_{n}\right|^{2 \alpha}+1\right)+C_{7}\left(\int_{0}^{T}\left|\dot{u}_{n}(t)\right|^{2} d t\right)^{\frac{\alpha+1}{2}} \\
& +C_{8}\left(\int_{0}^{T}\left|\dot{u}_{n}(t)\right|^{2} d t\right)^{\frac{1}{2}}-\int_{0}^{T} F\left(t, P \bar{u}_{n}\right) d t+\left\|\widetilde{u}_{n}\right\|_{\infty} \int_{0}^{T}|e(t)| d t \\
\leq & \frac{3}{4} \int_{0}^{T}\left|\dot{u}_{n}(t)\right|^{2} d t+C_{6}\left(\left|\bar{u}_{n}\right|^{2 \alpha}+1\right)+C_{7}\left(\int_{0}^{T}\left|\dot{u}_{n}(t)\right|^{2} d t\right)^{\frac{\alpha+1}{2}} \\
& +C_{10}\left(\int_{0}^{T}\left|\dot{u}_{n}(t)\right|^{2} d t\right)^{\frac{1}{2}}-\int_{0}^{T} F\left(t, P \bar{u}_{n}\right) d t
\end{aligned}
$$

for all large $n$ and some constants $C_{9}, C_{10}$. Then (10), (8) and $\left(5^{+}\right)$imply that $\left(\left|P \bar{u}_{n}\right|\right)$ is bounded. In fact, if not, without loss of generality we may assume that $\left|P \bar{u}_{n}\right| \rightarrow \infty$ as $n \rightarrow \infty$. Then from (8) and (10) one obtains

$$
\liminf _{n \rightarrow \infty}\left|P \bar{u}_{n}\right|^{-2 \alpha} \int_{0}^{T} F\left(t, P \bar{u}_{n}\right) d t>-\infty
$$

which contradicts $\left(5^{+}\right)$. Hence $\left(\left|P \bar{u}_{n}\right|\right)$ is bounded. Furthermore, $\left(\widetilde{u}_{n}\right)$ is bounded by (8), which implies that $\hat{u}_{n}$ is bounded. Arguing then as in Proposition 4.1 in [7], we conclude that the $(P S)$ condition is satisfied.

Now we check the link condition that

(a) $\inf \{\psi(\pi(u)) \mid \pi(u) \in Y \times V\}>-\infty$, and

(b) $\psi(\pi(x)) \rightarrow-\infty$ uniformly for $\pi(Q x) \in V$ as $|P x| \rightarrow \infty$, where $x \in R^{N}$.

For $\pi(u) \in Y \times V, u=Q \bar{u}+\widetilde{u}$. It follows from (9) that

$$
\begin{aligned}
\left|\int_{0}^{T}[F(t, u(t))-F(t, 0)] d t\right| \leq & \frac{1}{4} \int_{0}^{T}|\dot{u}(t)|^{2} d t+C_{6}+C_{7}\left(\int_{0}^{T}|\dot{u}(t)|^{2} d t\right)^{\frac{\alpha+1}{2}} \\
& +C_{8}\left(\int_{0}^{T}|\dot{u}(t)|^{2} d t\right)^{\frac{1}{2}}
\end{aligned}
$$


for all $\pi(u) \in Y \times V$. Hence, by (4), Sobolev's inequality (6) and (11), we have

$$
\begin{aligned}
\psi(\pi(u))= & \psi(\pi(\widetilde{u}+Q \bar{u})) \\
= & \varphi(\widetilde{u}+Q \bar{u}) \\
= & \frac{1}{2} \int_{0}^{T}|\dot{u}(t)|^{2} d t-\int_{0}^{T} F(t, 0) d t-\int_{0}^{T}[F(t, \widetilde{u}(t)+Q \bar{u})-F(t, 0)] d t \\
& +\int_{0}^{T}(e(t), \widetilde{u}(t)+Q \bar{u}) d t \\
\geq & \frac{1}{2} \int_{0}^{T}|\dot{u}(t)|^{2} d t-\int_{0}^{T} F(t, 0) d t-\left(|Q \bar{u}|_{\infty}+\|\widetilde{u}\|_{\infty}\right) \int_{0}^{T}|e(t)| d t \\
& -C_{6}-C_{7}\left(\int_{0}^{T}|\dot{u}(t)|^{2}\right)^{\frac{\alpha+1}{2}}-C_{8}\left(\int_{0}^{T}|\dot{u}(t)|^{2}\right)^{\frac{1}{2}} \\
\geq & \frac{1}{2} \int_{0}^{T}|\dot{u}(t)|^{2} d t-C_{11}-C_{7}\left(\int_{0}^{T}|\dot{u}(t)|^{2}\right)^{\frac{\alpha+1}{2}}-C_{12}\left(\int_{0}^{T}|\dot{u}(t)|^{2}\right)^{\frac{1}{2}}
\end{aligned}
$$

for all $\pi(u) \in Y \times V$, which implies (a). It follows from (4) and (2) that

$$
\begin{aligned}
\psi(\pi(x)) & =\varphi(x) \\
& =\varphi(\hat{x}) \\
& =-\int_{0}^{T} F(t, P x+Q x) d t \\
& =-\int_{0}^{T} F(t, P x) d t-\int_{0}^{T} \int_{0}^{1}(\nabla F(t, P x+s Q x), Q x) d s d t \\
\leq & -\int_{0}^{T} F(t, P x) d t+\int_{0}^{T} f(t)|P x+Q x|^{\alpha}|Q x| d t+\int_{0}^{T} g(t)|Q x| d t \\
\leq & -\int_{0}^{T} F(t, P x) d t+C_{13}|P x|^{\alpha}+C_{14}
\end{aligned}
$$

for all $x \in R^{N}$ and some constants $C_{13}$ and $C_{14}$. Hence we have, by $\left(5^{+}\right)$,

$$
\limsup _{|P x| \rightarrow \infty}|P x|^{-2 \alpha} \psi(\pi(x)) \leq-\infty
$$

uniformly for all $Q x \in R^{r}$, which implies (b). It follows from the generalized saddle point theorem (Theorem 1.7 in [4]) that $\psi$ has at least $r+1$ critical points. Hence $\varphi$ has at least $r+1$ geometrically distinct critical points. Therefore, in this case, problem (1) has at least $r+1$ geometrically distinct solutions in $H_{T}^{1}$. 
In the case that $\left(5^{-}\right)$holds, the proof relies on Theorem 4.12 in [7]. By (4), (9) and Sobolev's inequality (6), we have

$$
\begin{aligned}
\varphi(u)= & \varphi(\hat{u}) \\
= & \frac{1}{2} \int_{0}^{T}|\dot{u}(t)|^{2} d t-\int_{0}^{T} F(t, P \bar{u}) d t+\int_{0}^{T}(e(t), Q \bar{u}+\widetilde{u}(t)) d t \\
& -\int_{0}^{T} \int_{0}^{1}(\nabla F(t, P \bar{u}+s(Q \bar{u}+\widetilde{u}(t))), Q \bar{u}+\widetilde{u}(t)) d s d t \\
\geq & \frac{1}{4} \int_{0}^{T}|\dot{u}(t)|^{2} d t-\int_{0}^{T} F(t, P \bar{u}) d t-\left(|Q \bar{u}|+\|\widetilde{u}\|_{\infty}\right) \int_{0}^{T}|e(t)| d t \\
& -C_{6}\left(|P \bar{u}|^{2 \alpha}+1\right)-C_{7}\left(\int_{0}^{T}|\dot{u}(t)|^{2} d t\right)^{\frac{\alpha+1}{2}}-C_{8}\left(\int_{0}^{T}|\dot{u}(t)|^{2} d t\right)^{\frac{1}{2}} \\
\geq & \frac{1}{4} \int_{0}^{T}|\dot{u}(t)|^{2} d t-\int_{0}^{T} F(t, P \bar{u}) d t-C_{15}\left(|P \bar{u}|^{2 \alpha}+1\right) \\
& -C_{7}\left(\int_{0}^{T}|\dot{u}(t)|^{2} d t\right)^{\frac{\alpha+1}{2}}-C_{16}\left(\int_{0}^{T}|\dot{u}(t)|^{2} d t\right)^{\frac{1}{2}}
\end{aligned}
$$

for all $u \in H_{T}^{1}$, which implies that $\varphi$ is bounded from below. Moreover, the functional $\varphi$ satisfies the $(P S)_{G}$ condition; that is, for every sequence $\left(u_{n}\right)$ in $H_{T}^{1}$ such that $\varphi\left(u_{n}\right)$ is bounded and $\varphi^{\prime}\left(u_{n}\right) \rightarrow 0$, the sequence $\pi\left(u_{n}\right)$ has a convergent subsequence (see Definition 4.2 in [7]). In fact, the boundedness of $\varphi\left(u_{n}\right),\left(5^{-}\right)$and (12) imply that $\left(\widetilde{u}_{n}\right)$ and $\left(P \bar{u}_{n}\right)$ are bounded. Hence $\left(\hat{u}_{n}\right)$ is bounded. As in the proof of Proposition 4.1 in [7], $\left(\hat{u}_{n}\right)$ has a convergent subsequence; so does $\pi\left(u_{n}\right)=\pi\left(\hat{u}_{n}\right)$. Now the Theorem in this case follows from Theorem 4.12 in [7].

\section{ACKNOWLEDGMENT}

The authors thank the referee for valuable comments.

\section{REFERENCES}

1. M. Willem, Oscillations forcés de systemes hamiltoniens, Public Sémin. Analyse non linéaire Univ. Besancon, 1981.

2. P. H. Rabinowitz, On a class of functionals invariant under a $Z^{n}$ action, Trans. Amer. Math. Soc., 1988, 310(1), 303-311. MR 89i:34057

3. K. C. Chang, On the periodic nonlinearity and the multiplicity of solutions, Nonlinear Analysis TMA, 1989, 13(5), 527-537. MR 90k:58036

4. J. Q. Liu, A generalized saddle point theorem, J. Differential Equations, 1989, 82, 372-385. MR 90k:58035

5. Xing-Ping $\mathrm{Wu}$, Periodic solutions for nonautonomous second-order systems with bounded nonlinearity, J. Math. Anal. Appl., 1999, 230(1), 135-141. MR 2000b:34071

6. Chun-Lei Tang, Periodic solutions for nonautonomous second order systems with sublinear nonlinearity, Proc. Amer. Math. Soc., 1998, 126(11), 3263-3270. MR 99a:34122

7. J. Mawhin and M. Willem, Critical point theory and Hamiltonian systems, Springer-Verlag, New York, 1989. MR 90e:58016

8. J. Mawhin, Nonlinear oscillations: one hundred years after Liapunov and Poincaré, Z. Angew. Math. Mech., 1993, 73, T54-T62. MR 94e:34039

9. J. Mawhin, Forced second-order conservative systems with periodic nonlinearity, Anal. Non Linéaire, Inst. Henri Poincaré, Special issue dedicated to J. J. Moreau, 1989, 415-434. MR 90i:58165 
10. A. Fonda and J. Mawhin, Critical point theory and multiple periodic solutions of conservative systems with periodic nonlinearity, Proc. Internat. Conf. Theory Applications Differential Equations, Columbus, Ohio, 1988, 1, Ohio Univ. Press, 1989, 298-304. MR 91d:58039

Department of Mathematics, Southwest Normal University, Chongqing 400715, PeoPLE'S RePublic of China

E-mail address: tangcl@swnu.edu.cn

Department of Mathematics, Southwest Normal University, Chongqing 400715, PeoPLE'S REPUBlic OF CHINA

E-mail address: wuxingping@eduwest.com 